\title{
PARÂMETROS CINÉTICOS DE MOAGEM DE ITABIRITOS COMPACTO E FRIÁVEL
}

\author{
D. B. MAZZINGHY', J. F. C. RUSSO', D. A. PIMENTEL \\ Anglo American - Minério de Ferro \\ douglas.mazzinghy@angloamerican.com¹; jose.russo@angloamerican.com² ; \\ danielle.pimentel@angloamerican.com ${ }^{3}$
}

Artigo submetido em novembro/2013 e aceito em dezembro/2013

DOI: http://dx.doi.org/10.15628/holos.2014.1747

\section{RESUMO}

Duas amostras de minério de ferro classificadas como itabiritos compacto e friável foram testadas com relação à moabilidade. Os testes foram realizados em um moinho de bolas em escala de laboratório com torquímetro para medição precisa da potência. Os parâmetros cinéticos das amostras foram determinados por meio do aplicativo Moly-Cop Tools 2.0. As simulações também foram realizadas com o mesmo aplicativo para previsão do comportamento do circuito de moagem com cada amostra estudada. Os resultados indicam que a amostra de itabirito friável possui maior consumo específico de energia, mesmo sendo uma amostra de granulometria mais fina que a amostra de itabirito compacto.

PALAVRAS-CHAVE: Moagem, Simulação, Itabiritos, Parâmetros de Quebra.

\section{KINETIC PARAMETERS OF GRINDING OF COMPACT AND FRIABLE ITABIRITES}

\section{ABSTRACT}

Two samples of iron ore classified as friable and compact itabirites were tested for their grindability. The tests were carried out in a ball mill on a laboratory scale with a torque device for accurate measurement of power. The kinetic parameters of the samples were determined by Moly-Cop Tools 2.0. Simulations were performed with
\end{abstract}

the same application for forecasting the behavior of the grinding circuit with each of the samples studied. The results indicate that the friable itabirite sample has high specific energy consumption when compared with itabirite compact, even with more fines in particle size distributions.

KEYWORDS: Grinding, Simulation, Itabirites, Breakage Parameters. 


\section{INTRODUÇÃO}

O Projeto Minas-Rio, de propriedade da AngloAmerican, em fase de implantação, prevê o beneficiamento de minérios itabiríticos classificados como compacto e friável. A usina de beneficiamento, localizada na cidade de Conceição do Mato Dentro no estado de Minas Gerais, possui dois circuitos de moagem primária. Cada circuito é composto por um moinho de bolas de 7,8 m de diâmetro por 12,6 m de comprimento e duas baterias de 8 hidrociclones de $800 \mathrm{~mm}$ de diâmetro. Cada moinho de bolas possui dois motores de $7.500 \mathrm{~kW}$, totalizando $15.000 \mathrm{~W}$ por circuito. $\mathrm{O}$ circuito de moagem é do tipo direto. $\mathrm{O}$ objetivo deste trabalho é avaliar os parâmetros de quebra destes dois tipos litológicos que irão alimentar a usina de beneficiamento.

\subsection{Modelo de Moagem}

O modelo do balanço populacional é muito utilizado para a simulação de circuitos de moagem. Este tipo de modelo considera todas as faixas granulométricas e não apenas uma única faixa como o modelo empírico de BOND (1952). A Equação 1 descreve o modelo do balanço populacional para a moagem em batelada (AUSTIN et al., 1984).

$$
\frac{d m_{i}(t)}{d t}=-S_{i} m_{i}(t)+\sum_{j=1}^{i-1} b_{i j} S_{j} m_{j}(t)^{\prime}, \quad i=1,2, \ldots n
$$

$m_{i}(t)=$ fração em massa de partículas contidas no intervalo de tamanho $i$ após o tempo $t$ de moagem;

$S_{i}=$ função seleção das partículas no intervalo de tamanho $i ;$

$b_{i j}=$ função quebra ou fração em massa de partículas no intervalo de tamanho $i$ produzidas pela quebra de partículas no intervalo de tamanho $j$.

Equação 2 expressa um modelo para descrever a função quebra acumulada $B_{i j}$ (AUSTIN et al., 1984). Os parâmetros $B_{0}, B_{1}, B_{2}$ correspondem as constantes dependentes das características do material.

$$
B_{i, j}=\beta_{0}\left(\frac{d_{i}}{d_{j+1}}\right)^{\beta_{1}}+\left(1-\beta_{0}\right)\left(\frac{d_{i}}{d_{j+1}}\right)^{\beta_{2}}, \quad 0 \leq \beta_{0} \leq 1
$$

No entanto, a Equação 3 exprime um modelo para descrever a função seleção $S_{i}$ (AUSTIN et al., 1984). Os parâmetros $\alpha_{0}, \alpha_{1}, \alpha_{2}, d_{\text {crit }}$ correspondem as constantes dependentes do material e das condições de moagem. 


$$
S_{i}=\frac{\alpha_{0}\left(d_{i}\right)^{\alpha_{1}}}{1+\left(\frac{d_{i}}{d_{c r i t}}\right)^{\alpha_{2}}}, \quad \alpha_{2} \geq 0
$$

Segundo HERBST \& FUERSTENAU (1980) a função seleção $S_{i}$ tem relação de proporcionalidade com a potência absorvida pelo moinho e a massa contida neste, conforme a Equação 4.

$$
S_{i}=S_{i}^{E}\left(\frac{P}{H}\right)
$$

$S_{i}=$ função seleção no intervalo de tamanho $i\left(\min ^{-1}\right)$;

$S_{i}^{E}=$ função seleção específica em energia (t/kWh);

$P=$ potência líquida demandada pelo moinho $(\mathrm{kW})$;

$H=$ massa de material contida no moinho $(\mathrm{t})$.

\subsection{Estimativa de Potência}

A potência do moinho pode ser estimada segundo as suas dimensões e as condições operacionais, conforme a Equação 5 proposta por HOGG \& FUERSTENAU (1972).

$$
P_{\text {liq }}=\eta P_{\text {inst }}=0,238 D^{3,5}\left(\frac{L}{D}\right) N_{c} \rho_{a p}\left(J-1,065 J^{2}\right) \operatorname{sen} \alpha
$$

$P_{\text {liq. }}=$ potência líquida $(\mathrm{kW})$;

$P_{\text {inst }}=$ potência instalada $(\mathrm{kW})=P_{\text {cons. }} / \eta$

$\eta=$ eficiência de transmissão elétrica e de potência, \%;

$D=$ diâmetro interno do moinho (pés);

$L=$ comprimento efetivo do moinho (pés);

$N_{c}=$ Fração da velocidade crítica: $N_{c r i t}=76,6 / D^{0,5}$;

$\rho_{a p}=$ densidade aparente da carga $\left(\mathrm{t} / \mathrm{m}^{3}\right)$;

$J=$ enchimento volumétrico (\%);

$\alpha=$ ângulo de levantamento da carga.

Este modelo foi expandido de sua formulação original para descrever independentemente a contribuição de cada componente da carga, bolas e polpa, para a potência total do moinho por SEPÚLVEDA (2004). A densidade aparente da carga $\rho_{a p}$ pode ser estimada conforme a Equação 6. 


$$
\rho_{a p}=\frac{\left(1-f_{v}\right) \rho_{b} J_{b}+\rho_{p} J_{p} f_{v} J_{b}+\rho_{p}\left(J-J_{b}\right)}{J}
$$

$f_{v}=$ volume (\%) de vazios intersticiais entre as bolas (tipicamente assume ser $40 \%$ do volume aparente ocupado pelas bolas);

$J_{b}=$ enchimento aparente de bolas (\%) (incluindo bolas, polpa e vazios intersticiais entre as bolas); $J_{p}=$ enchimento intersticial da polpa (\%), corresponde à fração intersticial de vazios disponíveis (entre a carga de bolas) na verdade ocupada pela polpa e partículas finas;

$\rho_{p}=$ peso específico da polpa $\left(\mathrm{t} / \mathrm{m}^{3}\right)$.

\subsection{Modelo de Classificação}

O modelo de PLITT (1976) foi desenvolvido segundo a análise estatística de trezentos ensaios experimentais. As equações do modelo são descritas a seguir.

\section{Pressão de alimentação do hidrociclone}

$$
H=a_{1}\left(\frac{Q^{1,46} \exp \left(-7,63 \phi+10,79 \phi^{2}\right)}{(D c)^{0,20} h^{0,15}(D i)^{0,51}(D o)^{1,65}(D u)^{0,53}}\right)
$$

$a_{1}=$ constante dependente da aplicação;

$D_{C}=$ diâmetro do hidrociclone (pol.);

$D i=$ diâmetro de entrada do hidrociclone (pol.);

Do $=\operatorname{vortex}($ pol. $)$

$D u=\operatorname{apex}($ pol.);

$h=$ altura do hidrociclone (pol.);

$H=$ pressão de alimentação do hidrociclone (pés de coluna de polpa);

$Q=$ vazão de alimentação $\left(\mathrm{m}^{3} / \mathrm{h}\right)$;

$\varphi=$ fração de sólidos em volume na alimentação.

$\underline{\text { Tamanho de corte corrigido, } \mathrm{d}_{50 c}}$

$$
d_{50 c}=a_{2}\left(\frac{(D c)^{0,44}(D i)^{0,58}(D o)^{1,91} \exp (11,12 \phi)}{(D u)^{0,80} h^{0,37} Q^{0,44}\left(\rho_{s}-1\right)^{0,5}}\right)
$$


$a_{2}=$ constante dependente da aplicação. $O$ tamanho de corte corrigido $d_{50 c}$ é definido como sendo um tamanho de partícula que deveria ser dividido em $50 \%$ indo para o underflow e $50 \%$ indo para o overflow baseado na curva de eficiência corrigida.

\section{$\underline{\text { Partição de Fluxo }}$}

$$
S=a_{3} \frac{h^{0,19}\left(\frac{D u}{D o}\right)^{2,64} \exp \left(-4,33 \phi+8,77 \phi^{2}\right)}{H^{0,54}(D c)^{0,38}}
$$

$S=$ carga circulante volumétrica (razão entre underflow e overflow);

$a_{3}=$ constante dependente da aplicação.

Eficiência de Classificação Corrigida

$$
E_{i}^{c}=1-\exp \left[-0,693\left(\frac{d_{i}}{d_{50 c}}\right)^{m}\right]
$$

O parâmetro $m$ de PLITT pode ser calculado como segue:

$$
m=\exp \left[a_{4}-1,58 \frac{S}{(S+1)}\right]\left[\frac{(D c)^{2} h}{Q}\right]^{0,15}
$$

$a_{4}=$ constante dependente da aplicação.

\section{Curto Circuito de Polpa}

O modelo de classificação do CIMM (GUTIÉRREZ \& SEPÚLVEDA, 1986) é uma variação do modelo de PLITT. Este modelo considera mais uma equação para descrever o curto circuito de polpa.

$$
B_{p f}=\lambda B_{p w}
$$

$B_{p f}=$ curto circuito para underflow;

$B_{p w}=$ curto circuito de água;

$\lambda=$ constante dependente da aplicação.

O curto circuito de água $B_{p w}$ pode ser descrito pela Equação 13. 


$$
B_{p w}=\frac{\frac{S}{(S+1)}-\varphi R_{s}^{c}}{1-\varphi\left[1-\lambda\left(1-R_{s}^{c}\right)\right]}
$$

$R_{s}{ }^{c}=$ recuperação total de sólidos em peso (hipotética).

1.4 Índice de Trabalho Operacional

O índice de trabalho operacional $W_{i o}$ é descrito conforme a Equação 14 (BOND, 1952).

$$
W_{i o}=\frac{E}{10\left[\left(\frac{1}{\sqrt{P_{80}}}\right)-\left(\frac{1}{\sqrt{F_{80}}}\right)\right]}
$$

$W_{i o}=$ Índice de trabalho operacional $(\mathrm{kWh} / \mathrm{t})$;

$E=$ Consumo específico de energia $(\mathrm{kWh} / \mathrm{t})$;

$F_{80}=80 \%$ passante na alimentação nova do circuito $(\mu \mathrm{m})$;

$P_{80}=80 \%$ passante no produto do circuito $(\mu \mathrm{m})$.

\section{METODOLOGIA}

\subsection{Amostras}

Na Figura 1 consta as distribuições granulométricas das amostras de itabiritos compacto e friável.

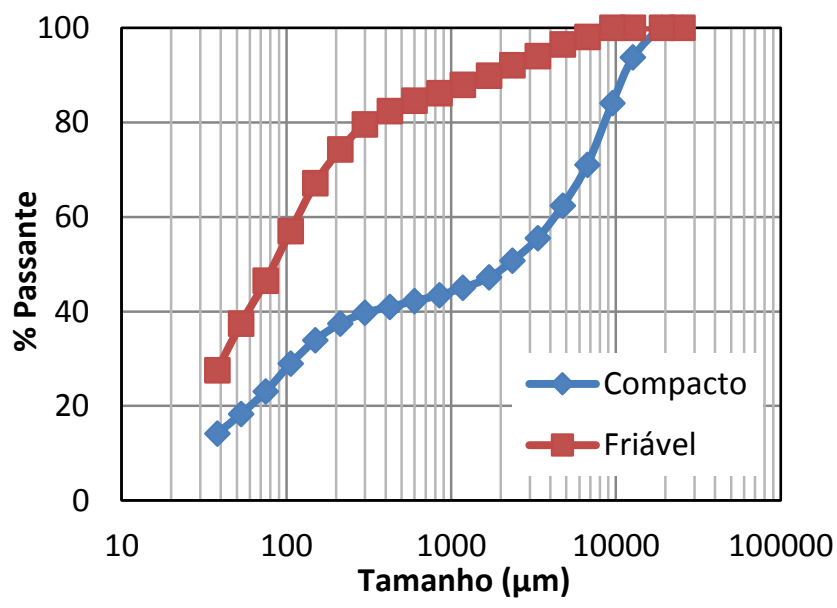

Figura 1 - Distribuição granulométrica das amostras de itabirito compacto e friável 


\subsection{Carga Moedora}

A distribuição de tamanhos das bolas utilizadas nos testes com as amostras de itabiritos compacto e friável está descrita na Tabela I.

Tabela I - Distribuição de tamanhos das bolas utilizadas nos testes

\begin{tabular}{cc}
\hline $\mathrm{mm}$ & $\%$ \\
\hline 63,5 & 36,0 \\
50,8 & 41,0 \\
38,1 & 17,2 \\
25,4 & 5,8 \\
Total & 100 \\
\hline
\end{tabular}

\subsection{Moinho com Medição de Torque}

Os testes foram realizados em um moinho de bolas em escala de laboratório com 0,46 m de diâmetro por 0,38 $\mathrm{m}$ de comprimento. 0 moinho possui um torquímetro para determinação precisa da potência requerida nos testes. As amostras foram moídas em diferentes tempos de forma a obter curvas granulométricas que possibilitem a determinação dos parâmetros cinéticos das amostras estudadas. Os testes foram realizados com 30\% de enchimento de bolas e com $75 \%$ da velocidade crítica, inclusive com $70 \%$ de sólidos em peso na polpa.

\subsection{Parâmetros Cinéticos de Moagem}

Os parâmetros cinéticos foram determinados com auxílio do aplicativo Moly-Cop Tools 2.0 utilizando os resultados dos testes com moinho de bolas. $\mathrm{O}$ aplicativo realiza a minimização dos quadrados de uma função objetivo até que o modelo expresse de forma aceitável os dados experimentais. Caso o modelo não represente as curvas granulométrica deve-se verificar a possibilidade de erro nos dados experimentais utilizados.

\subsection{Parâmetros de Classificação}

Para determinar os parâmetros de classificação é necessário fazer testes com hidrociclones de mesmo diâmetro que os hidrociclones que serão simulados. Assim, por falta de testes com hidrociclones de $813 \mathrm{~mm}$ de diâmetro, foram adotados valores de referência para os parâmetros de classificação. Sabe-se que as amostras, provavelmente, possuem diferentes parâmetros de classificação. Neste trabalho, adotou-se o mesmo conjunto de parâmetros de classificação para as duas amostras estudadas por se tratar de uma avaliação inicial de comparação entre os minérios com relação à moabilidade. Estudos futuros devem ser realizados com os modelos de classificação calibrados com testes em hidrociclones de grande diâmetro. 


\section{3. RESULTADOS E DISCUSSÃO}

\subsection{Parâmetros Cinéticos de Moagem}

Os parâmetros cinéticos das amostras estudadas estão relacionados na Tabela II.

Tabela II - Parâmetros cinéticos das amostras estudadas

\begin{tabular}{cccccccc}
\hline Função & \multicolumn{4}{c}{ Seleção } & \multicolumn{3}{c}{ Quebra } \\
\hline Parâmetros & $\alpha_{0}(\mathrm{a})$ & $\alpha_{1}(\alpha)$ & $\alpha_{2}(\Lambda)$ & $D_{\text {crit }}(\mu)$ & $\beta_{0}(\phi)$ & $\beta_{1}(\lambda)$ & $\beta_{2}(\beta)$ \\
\hline Compacto & 0,00039 & 1,413 & 2,50 & 1641,9 & 0,145 & 0,496 & 4,00 \\
Friável & 0,00003 & 1,725 & 2,50 & 6579,6 & 0,200 & 0,250 & 4,00 \\
\hline
\end{tabular}

O aplicativo Moly-Cop Tools 2.0 utiliza diferentes letras para nomear os parâmetros das funções seleção e quebra. As letras normalmente utilizadas para descrever estes parâmetros estão entre parênteses, Tabela 3 (AUSTIN et al., 1984).

\subsection{Parâmetros de Classificação}

Os parâmetros de classificação adotados nas simulações e as condições operacionais dos hidrociclones industriais estão relacionados na Tabela III e IV.

Tabela III - Parâmetros de classificação utilizados para simulação das amostras estudadas

\begin{tabular}{ccccc}
\hline $\boldsymbol{a}_{1}$ & $\boldsymbol{a}_{\mathbf{2}}$ & $\boldsymbol{a}_{\mathbf{3}}$ & $\boldsymbol{a}_{\mathbf{4}}$ & $\boldsymbol{\lambda}$ \\
\hline 10,14 & 1,33 & 51,44 & 0,39 & 0,95 \\
\hline
\end{tabular}

Tabela IV - Condições operacionais dos hidrociclones industriais

\begin{tabular}{ccccccc}
\hline Hidrociclones & Diâmetro & Altura & Entrada & Vortex & Apex & Pressão \\
\hline (Quant.) & $(\mathrm{mm})$ & $(\mathrm{mm})$ & $(\mathrm{mm})$ & $(\mathrm{mm})$ & $(\mathrm{mm})$ & $\left(\mathrm{kgf} / \mathrm{cm}^{2}\right)$ \\
\hline 16 & 813 & 2438 & 203 & 284 & 156 & 1,25 \\
\hline
\end{tabular}

\subsection{Simulações}

O modelo de moagem foi alimentado com os parâmetros determinados nos testes com moinho de bolas em escala de laboratório. O moinho em escala industrial possui 7,8 $\mathrm{m}$ de diâmetro e 12,6 m de comprimento e será operado com $75 \%$ da velocidade crítica. Na Figura 2 consta a representação gráfica da função seleção específica e da função quebra das amostras estudadas. 

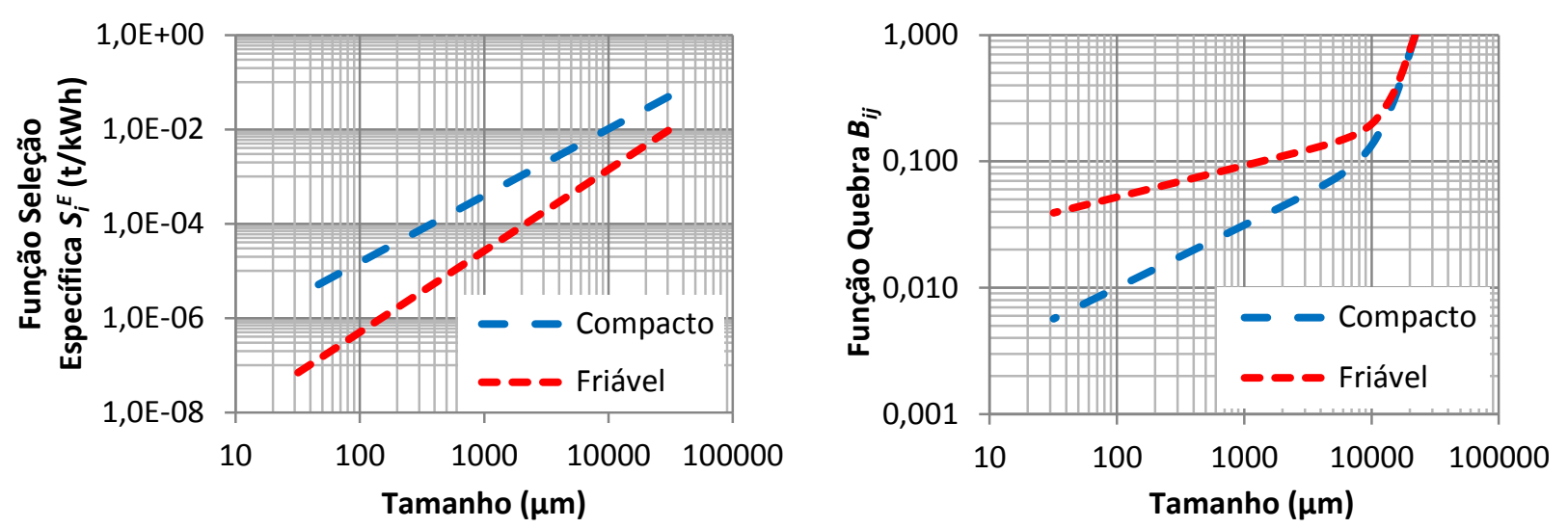

Figura 2 - Função seleção e função quebra das amostras de itabirito compacto e friável.

A função seleção específica das duas amostras testadas é do tipo normal, ou seja, a taxa de quebra cresce com o aumento do tamanho das partículas. Quando há diferença significativa entre o tamanho das bolas e o maior tamanho de partícula, a função seleção pode ser do tipo anormal, que significa dizer que a taxa de quebra diminui desde um determinado tamanho. A amostra de itabirito compacto possui uma função seleção específica maior ou maior taxa de quebra por unidade de energia ( $\mathrm{t} / \mathrm{kWh}$ ). Assim, espera-se que o consumo energético seja menor devido à maior eficiência (taxa de quebra) desta amostra. As curvas de função quebra, mais horizontalizadas, demonstram que as amostras testadas têm a propriedade de geração de finos após os eventos de quebra. A função quebra da amostra de itabirito friável indica que esta amostra gera mais finos que a amostra de itabirito compacto.

\subsection{Avaliação do Índice de Trabalho Operacional (Wio)}

O índice de trabalho operacional $W_{i o}$ foi determinado para as duas amostras estudadas. $\mathrm{Na}$ Tabela $V$ incluem-se os resultados obtidos nas simulações. A análise dos resultados obtidos com amostra de itabirito friável revelou elevado consumo específico de energia, mesmo com um $F_{80}$ bem inferior ao da amostra de itabirito compacto. Segundo TURRER et al. (2013), a amostra de itabirito friável possui grãos de quartzo maiores que a amostra de itabirito compacto. Esta diferença poderia justificar o maior consumo energético da amostra de itabirito friável.

Tabela V - Índice de Trabalho Operacional para as amostras de itabirito compacto e friável

\begin{tabular}{ccc}
\hline Amostra & Compacto & Friável \\
\hline$F_{80}(\mu \mathrm{m})$ & 8582 & 317 \\
$P_{80}(\mu \mathrm{m})$ & 113 & 105 \\
$W_{i 0}(\mathrm{kWh} / \mathrm{t})$ & 5,85 & 11,74 \\
\hline
\end{tabular}

\section{CONCLUSÃO}

Segundo a análise dos resultados, pode-se afirmar que a moagem da amostra de itabirito friável requer um consumo específico de energia maior, em relação ao da amostra de itabirito compacto. Esperava-se que o consumo energético da amostra de itabirito friável fosse menor, uma 
vez que esta amostra possui maior quantidade de finos. A amostra de itabirito friável possui grãos de quartzo maiores e a hematita está incrustada nestes grãos, diferente da amostra de itabirito compacto. Estudos mais detalhados se fazem necessários para investigar os resultados obtidos.

Parabéns aos autores pela redação e exposição dos dados.

\section{REFERÊNCIAS BIBLIOGRÁFICAS}

1. AUSTIN, L. G., KLIMPEL, R. R., LUCKIE, P. T. Process Engineering of Size Reduction, SME - AIME, 1984.

2. BOND, F. C. The Third Theory of Comminution, AIME Trans.,Vol. 193, pp. 484, 1952

3. GUTIÉRREZ, L., SEPÚLVEDA, J. E. Dimensionamiento y Optimización de Plantas Concentradoras mediante Técnicas de Modelación Matemática, Publicación CIMM - Centro de Investigacion Minera y Metalúrgica, Santiago, Chile, 1986.

4. HERBST, J. A. \& FUERSTENAU, D. W. Scale-Up Procedure for Continuous Grinding Mill Design Using Population Balance Models, International Journal of Mineral Processing, Vol. 7, pp. 131, 1980.

5. HOGG, R. \& FUERSTENAU, D. W. Power Relations for Tumbling Mills, Trans. SME-AIME, Vol. 252, pp. 418-432, 1972.

6. PLITT, L. R. A Mathematical Model for the Hydrocyclone Classifier, CIM Bulletin, pp. 114, December, 1976.

7. SEPÚLVEDA, J. E. Methodologies for the Evaluation of Grinding Media Consumption Rates at Full Plant Scale, Minerals Engineering, 17, pp. 1269-1279, 2004.

8. TURRER, H.D.G, SAWASATO, E.Y., GUIMARÃES, F.R., MAGALHÃES, C.R., DIAS, G.S., RUSSO, J.C., PIMENTEL, D.A., MAZZINGHY, D.B. Avaliação do consumo energético na moagem de dois diferentes tipos litológicos da Serra do Sapo, Submetido: $43^{\circ}$ Seminário de Redução de Minério de Ferro e Matérias-Primas - ABM, Belo Horizonte, 2013. 